\title{
Improving Accuracy and Diversity in Matching of Recommendation with Diversified Preference Network
}

\author{
Ruobing Xie* \\ WeChat, Tencent \\ Beijing, China \\ ruobingxie@tencent.com \\ Ziwei Zhang \\ Tsinghua University \\ Beijing, China \\ zw-zhang16@mails.tsinghua.edu.cn
}

\author{
Qi Liu* \\ WeChat, Tencent \\ Beijing, China \\ addisliu@tencent.com \\ Peng Cui \\ Tsinghua University \\ Beijing, China \\ cuip@tsinghua.edu.cn \\ Leyu Lin \\ WeChat, Tencent \\ Beijing, China \\ goshawklin@tencent.com
}

\author{
Shukai Liu \\ WeChat, Tencent \\ Beijing, China \\ shukailiu@tencent.com \\ Bo Zhang \\ WeChat, Tencent \\ Beijing, China \\ nevinzhang@tencent.com
}

\begin{abstract}
Recently, real-world recommendation systems need to deal with millions of candidates. It is extremely challenging to conduct sophisticated end-to-end algorithms on the entire corpus due to the tremendous computation costs. Therefore, conventional recommendation systems usually contain two modules. The matching module focuses on the coverage, which aims to efficiently retrieve hundreds of items from large corpora, while the ranking module generates specific ranks for these items. Recommendation diversity is an essential factor that impacts user experience. Most efforts have explored recommendation diversity in ranking, while the matching module should take more responsibility for diversity. In this paper, we propose a novel Heterogeneous graph neural network framework for diversified recommendation (GraphDR) in matching to improve both recommendation accuracy and diversity. Specifically, GraphDR builds a huge heterogeneous preference network to record different types of user preferences, and conduct a fieldlevel heterogeneous graph attention network for node aggregation. We also innovatively conduct a neighbor-similarity based loss to balance both recommendation accuracy and diversity for the diversified matching task. In experiments, we conduct extensive online and offline evaluations on a real-world recommendation system with various accuracy and diversity metrics and achieve significant improvements. We also conduct model analyses and case study for a better understanding of our model. Moreover, GraphDR has been deployed on a well-known recommendation system, which affects millions of users. The source code will be released.
\end{abstract}

${ }^{*}$ Both authors contributed equally to this research.

Permission to make digital or hard copies of part or all of this work for personal or classroom use is granted without fee provided that copies are not made or distributed for profit or commercial advantage and that copies bear this notice and the full citation on the first page. Copyrights for third-party components of this work must be honored.

For all other uses, contact the owner/author(s).

arXiv '21, Feb 07, 2021, Online

(c) 2021 Copyright held by the owner/author(s)

ACM ISBN 978-x-xxxx-xxxx-x/YY/MM.

https://doi.org/10.1145/xxxxxxx.Xxxxxxx

\section{CCS CONCEPTS}

- Information systems $\rightarrow$ Recommender systems; • Computing methodologies $\rightarrow$ Neural networks.

\section{KEYWORDS}

recommender system, diversified recommendation, graph neural network, heterogeneous network

\section{ACM Reference Format:}

Ruobing Xie, Qi Liu, Shukai Liu, Ziwei Zhang, Peng Cui, Bo Zhang, and Leyu Lin. 2021. Improving Accuracy and Diversity in Matching of Recommendation with Diversified Preference Network. In $\operatorname{arXiv}$ '21, Feb 07, 2021, Online. ACM, New York, NY, USA, 11 pages. https://doi.org/10.1145/xxxxxxx. $\operatorname{xxxxxxx}$

\section{INTRODUCTION}

Recently, real-world personalized recommendation systems usually need to deal with hundreds of millions of items [39]. Therefore, it is challenging to conduct complicated end-to-end recommendation algorithms on the entire corpus, for even a linear time complexity w.r.t the corpus size is unacceptable [54]. To balance both effectiveness and efficiency in real-world scenarios, conventional recommendation systems usually consist of two modules, namely the matching module and the ranking module $[6,47]$. The matching module, also regarded as the candidate generation in the Youtube model [6], aims to retrieve a small subset of (usually hundreds of) items from the entire corpus efficiently. In contrast, the ranking module conducts sophisticated models on these retrieved items to get specific item ranks. Fig. 1 shows the classical two-step architecture. The matching module concentrates more on the diversity, efficiency and item coverage, while the ranking module focuses more on the accuracy of specific item ranks. This two-step architecture balances efficiency and effectiveness in practice.

Conventional recommendation models usually regard recommendation accuracy metrics such like Click-through-rate (CTR) as their central objectives, in which popular items clicked by users are 


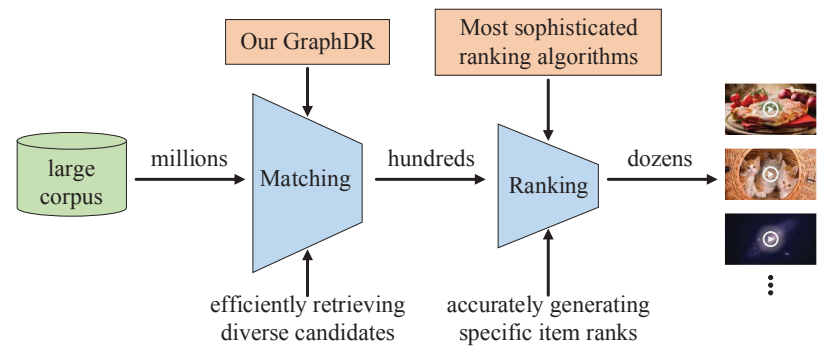

Figure 1: An example of a real-world recommendation system. GraphDR focuses on the matching module, which aims to retrieve user-interested and diverse items efficiently.

more preferred. However, such objectives will lead to homogenization issues that reduce personalization and harm user experiences. To solve this issue, recommendation diversity is considered to evaluate the overall recommendation performances from another aspect [2]. It is measured in two classical ways: the individual diversity and the aggregate diversity [21]. The individual diversity focuses on the local diversity in each recommended item list, which aims to balance user-item similarities and item-item dissimilarities [3]. In contrast, the aggregate diversity focuses on the global diversity in the overall recommendation, which is usually measured by the coverage of items that could be recommended by models in the entire corpus [16]. The significance of diversity has been widely verified to provide highly idiosyncratic items in recommendation [49], which should be considered in real-world scenarios.

There are lots of ranking models that have explored recommendation diversities with the help of dissimilarity factors [2], external taxonomy information [57], clustering [1] and graphic technologies [29]. However, most diversified recommendation models are specially designed for ranking, which are incredibly time-consuming to be used in matching with millions of items [29], while very few works systematically focus on the diversity in matching. In fact, matching should take more responsibility for diversity, since it cares more about the coverage of user-interested items rather than their specific item ranks. The recommendation diversity needs to be first guaranteed in the matching module. Otherwise, the homogenization of the item candidates generated by the matching module will inevitably lead to the lack of diversity in the final recommendation.

In this paper, we aim to improve both recommendation accuracy and diversity in the matching module, which is essential in realworld recommendation systems. We propose a novel Heterogeneous graph neural network framework for diversified recommendation (GraphDR). Precisely, GraphDR mainly consists of three modules: (1) Diversified preference network construction, which aims to build a huge global heterogeneous network containing various interactions between different types of nodes including videos, tags, medias, users and words. These interactions between essential recommendation factors reflect user diverse preferences from a global view, which are the sources of diversity. (2) Heterogeneous network representation learning (NRL), which learns node representations with a novel field-level heterogeneous graph attention network (FH-GAT). FH-GAT helps to better maintain and aggregate different types of interactions. We also innovatively conduct a neighbor-similarity based objective to encode user diverse preferences into heterogeneous node representations. Different from CTR-oriented objectives that simply focus on click behaviors, the neighbor-similarity based objective highlights diversity by considering multiple factors of videos such as user watching habit, audience community, video content, video taxonomy, and content provider. (3) Online multi-channel matching, which generates a small subset of user-interested and diverse item candidates efficiently through multiple channels. The multi-channel strategy is conducted to further amplify the diversity in the final results. The diversity derives from all three modules in GraphDR.

In experiments, we conduct both offline and online evaluations on a real-world video recommendation system, which is widely used by hundreds of millions of users. We conduct extensive experiments to measure the recommendation accuracy and diversity with dozens of metrics. We also explore GraphDR with model analyses, ablation tests and case studies for better understanding. The main contributions are concluded as follows:

- We highlight and systematically explore the recommendation diversity issue in the matching module, which is essential in practical large-scale recommendation systems.

- We propose a novel GraphDR framework to jointly improve both recommendation accuracy and diversity in real-world matching. To the best of our knowledge, we are the first to introduce GNN on heterogeneous preference networks for diversified recommendation in matching.

- We propose a novel field-level heterogeneous GAT model to aggregate neighbors with different feature fields. We also innovatively conduct the neighbor-similarity based loss to polish recommendation diversity.

- The offline and online evaluations indicate that GraphDR can improve both accuracy and diversity in practice. GraphDR is simple and effective, which has been deployed on a realworld recommendation system used by millions of users. It is also convenient to adopt GraphDR to other scenarios.

\section{RELATED WORKS}

In related works, we first give a brief introduction to the classical recommendation algorithms, and then introduce the efforts in recommendation diversity. We also include a discussion on the graph neural networks used in recommendation.

\subsection{Recommendation Systems}

Collaborative filtering $(\mathrm{CF})$ is a classical method which recommends items with similar items or users [34]. Matrix factorization (MF) attempts to decompose user-item interaction matrix to get user and item representations [20]. FM [33] expands to model second-order feature interactions with latent vectors. However, most neural ranking models rely on user-item interactions for prediction. Hence, these complicated ranking models are hard to be directly used in matching, for they are extremely time-consuming when handling million-level items. With the thriving in deep learning, neural models like Deep Crossing [35], FNN [51], PNN [32], Wide\&Deep [4], DCN [40] and DFN [46] are proposed to improve recommendation performances. DeepFM [10], AFM [45] and NFM [12] improve the 
original FM with DNN or attention. AutoInt [36] and BERT4Rec [37] also brings in self attention. Recently, AFN [5] and AutoFIS [23] are proposed to smartly model high-order feature interactions via logarithmic transformation or automatic feature selection. Most deep ranking models are challenging to be utilized in real-world matching module, for they are extremely time-consuming dealing with millions of candidates.

In contrast, there are much fewer works specially designed for matching. Conventional systems usually use IR-based methods [17] or Collaborative filtering (CF) based methods [34] for fast retrieval. For neural models, embedding-based retrieval such as DSSM [15] is also widely deployed. Recently, Youtube [6] brings in deep models to learn user preference in matching. Moreover, TDM [54], JTM [53] and OTM [56] arrange items with tree structures to accelerate top-n item retrieval, which combine matching and ranking in a single model. ICAN [47] is specially designed for cold-start multi-channel matching. Huang et al. [14] also proposes an industrial embedding-based retrieval framework in Facebook search. However, these matching models mainly focus on CTRoriented objectives. It is still challenging for these models to balance accuracy and diversity in real-world scenarios. In this work, we aim to improve both recommendation accuracy and diversity in the matching module via the proposed GraphDR with the diversified preference network.

\subsection{Diversified Recommendation}

Merely using CTR-oriented objectives will make hot items hotter, which inevitably brings in serious homogenization issues that may degrade user experiences [50]. The significance of diversity has been verified by lots of efforts, since it could provide highly idiosyncratic items with less homogeneity for users in personalized recommendation $[2,49]$. Recommendation diversity is mainly measured in individual diversity and aggregate diversity [21]. The individual diversity focuses on the local diversity in recommended list. [2] and [57] focus on intra-list item dissimilarities. [50] proposes a novel item novelty, which measures the additional information from new items. Some works measure diversity with the varieties of taxonomy in item lists [57]. In contrast, the aggregate diversity measures the global diversity in overall systems. [16] measures this diversity with the coverage of recommended items. The higher item coverage indicates that the model could recommend more long-tail items, which implies a more diversified system from the global aspect.

There are some works that model diversity in ranking. [2] bring dissimilarity factors to the loss functions to measure the individual diversity. External taxonomy information (e.g., tag, category and subtopic) [42, 57] and knowledge graph [9] are useful factors for diversity. Other technologies such as entropy regularizer [31], clustering [1], graph-based models [28, 29, 55], and greedy map inference [3] have also been explored for diversified recommendation. Recently, diversified recommendation is armed with reinforcement learning [25] and adversarial learning [43]. Recommendation bandits [22, 27] are also well explored. However, most diversified models are specially designed for ranking, which are hard to be directly used in matching. To the best of our knowledge, we are the first to use GNN on the global heterogeneous interactions to improve both accuracy and diversity in the matching module.

\subsection{Graph Neural Network (GNN)}

Recently, GNN has been widely explored and verified in various fields. GCN [19] introduces convolution to graphs based on spectral graph theory. GraphSAGE [11] conducts an inductive representation learning on large graphs. Graph attention network (GAT) [38] brings in graph attention mechanism. HetGNN [48] and HAN [41] extend GNN to heterogeneous networks. In recommendation, $\mathrm{Wu}$ et al. [44], Fan et al. [8] and He et al. [13] further use GNN on session-based and social-based recommendation. Heterogeneous graphs are also widely adopted to model different types of essential objects such as users, items, tags and providers in recommendation $[24,26]$. Inspired by these models, we conduct a heterogeneous graph to model various types of feature interactions, and also use a heterogeneous GNN model for node aggregation.

\section{METHODOLOGY}

In this paper, we propose GraphDR to improve both accuracy and diversity in matching by considering user diverse preferences. In this section, we first show the overall framework of GraphDR (Sec. 3.1). Second, we introduce the construction of nodes and edges in the diversified preference network, which is the source of diversity in our model (Sec. 3.2). Next, we introduce the Diversity-enhanced network representation learning model FH-GAT used to generate node representations for all types of nodes (Sec. 3.3). Finally, we give a detailed discussion on the proposed Diversity-enhanced training objective (Sec. 3.4). We further introduce the online deployment of the multi-channel matching module (Sec. 4).

\subsection{Overall Architecture}

The GraphDR framework mainly contains three modules as in Fig 2 , including diversified preference network construction, network representation learning, and online multi-channel matching. In offline NRL, GraphDR first collects various informative interactions between heterogeneous nodes to build a huge global diversified preference network. Next, we propose a field-level HGAT model to learn node embeddings with the neighbor-similarity based objective. In online serving, the multi-channel matching retrieves hundreds of accurate and diverse item candidates efficiently with multiple channels. The offline NRL conducts time-consuming training to encode user diverse preferences into node embeddings, while the online serving efficiently uses these learned embeddings for fast and diversified multi-channel retrieval.

\subsection{Diversified Preference Network}

The diversified preference network is the fundamental of diversity. We attempt to bring in heterogeneous interactions between essential objects in recommendation to describe user diverse preferences. Precisely, we focus on five different types of nodes including video, tag, media, user and word, which are essential factors that may impact users in recommendation. Each video has a title (containing words) and several tags annotated by editors. The video provider is viewed as the media. To alleviate the data sparsity and reduce computation costs, we cluster users into user groups as communities according to their basic profiles (i.e., the gender-age-location attribute triplets in this work), and consider these user groups as user nodes. We group users via user basic profiles for higher coverage. 


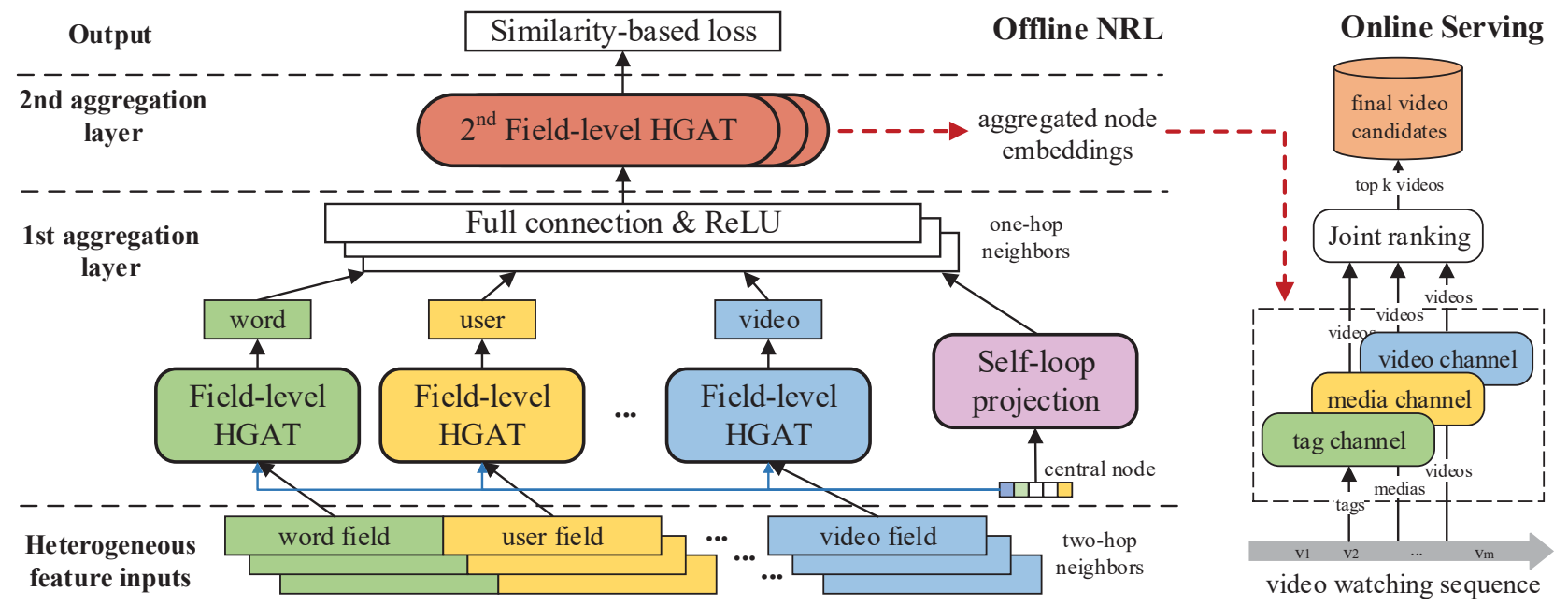

Figure 2: The offline NRL and online serving parts of GraphDR for matching in recommendation. The left offline NRL part is the proposed FH-GAT model, which builds the aggregated node embeddings with heterogeneous GAT on the diversified preference network. The right online multi-channel matching part aims to retrieve hundreds of videos from large corpora efficiently. The recommendation diversity comes from diversified preference network, FH-GAT trained with diversity-enhanced training objective, and online multi-channel matching.

We assume that the interactions between these five types of objects can reflect user diverse preferences. In GraphDR, we consider six types of edges to record these multi-aspect preferences as:

- Video-video edge. We generate the video-video edge between two video nodes if they have appeared adjacently in a user's video session. To reduce noises, we only use the valid watching behaviors, where videos have been watched for more than $70 \%$ of their total time lengths. Video-video edges record the sequential user watching habits in sessions.

- Video-user edge. Video-user edges are built if a video is validly watched by a user group at least 3 times in a week. This edge stores coarse-grained user-item interactions and also implies the audience community of videos.

- Video-tag edge. Video-tag edge connects videos with their corresponding tags, which reflects the coarse-grained semantic preferences of taxonomy in videos.

- Video-word edge. Video-word edge links videos with their words in titles, which reflects the fine-grained semantic preferences of detailed word-level contents in videos.

- Video-media edge. Video-media edges are drawn between videos and their medias, which shows the video providers.

- Tag-tag edge. We build tag-tag edges according to tag cooccurrence in a video, which highlights taxonomy relevance.

All edges are undirected and unweighted. These heterogeneous edges bring in additional information of videos besides user-item click behaviors. They can reflect user diverse preferences in user watching habit, audience community, video content, taxonomy, and content provider. For instance, two related videos may be linked via the same user groups, video providers, tags or watching sessions, or even connected by a multi-step path containing heterogenous nodes. The multi-hop paths via heterogeneous nodes and edges build up the potential reasons for recommendation, which are implicit, lowcorrelational but diversified. It is also not difficult to extend other types of nodes and edges in GraphDR.

\subsection{Diversity-enhanced Network Representation Learning}

Network representation learning aims to encode user diverse preferences into node representations. Inspired by [24, 41], we propose a new Field-level Heterogeneous Graph Attention Network (FH-GAT). Fig. 2 shows the 2-layer architecture.

3.3.1 Heterogeneous Feature Layer. We first project all heterogeneous nodes into the same feature space. For the k-th node, its overall neighbor set $N_{k}$ could be divided into five feature fields according to their types as $\left\{\overline{\boldsymbol{v}}_{k}, \overline{\boldsymbol{t}}_{k}, \overline{\boldsymbol{m}}_{k}, \overline{\boldsymbol{u}}_{k}, \overline{\boldsymbol{d}}_{k}\right\}$, where $\overline{\boldsymbol{v}}_{k}, \overline{\boldsymbol{t}}_{k}, \overline{\boldsymbol{m}}_{k}$, $\overline{\boldsymbol{u}}_{k}$ and $\overline{\boldsymbol{d}}_{k}$ indicate the one-hot representations of video, tag, media, user, word neighbors respectively. The node feature embeddings of the $\mathrm{k}$-th node $\boldsymbol{h}_{k}$ is as follows:

$$
f_{k}=\operatorname{concat}\left(\boldsymbol{v}_{k}, \boldsymbol{t}_{k}, \boldsymbol{m}_{k}, \boldsymbol{u}_{k}, \boldsymbol{d}_{k}\right),
$$

in which $v_{k}$ indicates the video-field feature embedding. In this work, we empirically set $\boldsymbol{v}_{k}=\boldsymbol{P}_{v} \overline{\boldsymbol{v}}_{k}$, where $\boldsymbol{P}_{v} \in \mathbb{R}^{d_{v} \times n_{v}}$ represents the lookup projection matrix generating $v_{k}$ with the video neighbors. $d_{v}$ is the dimension of $v_{k}$ and $n_{v}$ is the number of video nodes. For efficiency, the projection matrix is pre-defined as the indicator of top-frequent video neighbors and fixed during training. concat $(\cdot)$ is the concatenation operation. The tag, media, user and word field feature embeddings $\boldsymbol{t}_{k}, \boldsymbol{m}_{k}, \boldsymbol{u}_{k}$ and $\boldsymbol{d}_{k}$ are generated similarly as the video field feature embedding $v_{k}$.

3.3.2 Field-level HGAT Layer. This layer takes the neighbor feature embeddings $\left\{f_{1}, \cdots, f_{l}\right\}$ of the k-th node as inputs. We set a weighting vector group $\left\{\boldsymbol{w}_{k}^{v}, \boldsymbol{w}_{k}^{t}, \boldsymbol{w}_{k}^{m}, \boldsymbol{w}_{k}^{u}, \boldsymbol{w}_{k}^{d}\right\}$ for each field, where 
$\boldsymbol{w}_{k}^{v}$ represents the k-th weighting vector of video. The output embedding $\boldsymbol{y}_{k}^{v}$ of the video field is defined as follows:

$$
\boldsymbol{y}_{k}^{v}=\sum_{i=1}^{l} \alpha_{k i}^{v} \boldsymbol{v}_{i}, \quad \alpha_{k i}^{v}=\frac{\exp \left(\boldsymbol{w}_{k}^{v \top} \boldsymbol{v}_{i}\right)}{\sum_{j=1}^{n} \exp \left(\boldsymbol{w}_{k}^{v \top} \boldsymbol{v}_{j}\right)},
$$

where $\alpha_{k i}^{v}$ is the weight of the k-th node to its $\mathrm{i}$-th neighbor in the video field. The construction of $\boldsymbol{y}_{k}^{t}, \boldsymbol{y}_{k}^{m}, \boldsymbol{y}_{k}^{u}$ and $\boldsymbol{y}_{k}^{d}$ are the same as $\boldsymbol{y}_{k}^{v}$ We concatenate these embeddings to form the final neighbor-based representation $\boldsymbol{y}_{k}^{N}$ as follows:

$$
\boldsymbol{y}_{k}^{N}=\operatorname{ReLU}\left(\boldsymbol{W}_{\boldsymbol{n}} \cdot \operatorname{concat}\left(\boldsymbol{y}_{k}^{v}, \boldsymbol{y}_{k}^{t}, \boldsymbol{y}_{k}^{m}, \boldsymbol{y}_{k}^{u}, \boldsymbol{y}_{k}^{d}\right)\right) \text {. }
$$

We further consider the self-loop projection as a supplement to highlight the central k-th node's information. We have:

$$
\boldsymbol{y}_{k}^{S}=\operatorname{ReLU}\left(\boldsymbol{W}_{s} \cdot \boldsymbol{f}_{k}\right) \text {. }
$$

Next, we combine neighbor and self-loop based representations to get the 1st layer output $\boldsymbol{y}_{k}$, and use the 2nd FH-GAT layer to get the final aggregated representation $\boldsymbol{h}_{k}$ as:

$$
\boldsymbol{h}_{k}=\mathrm{FH}-\mathrm{GAT}\left(\boldsymbol{y}_{k}\right), \quad \boldsymbol{y}_{k}=\lambda_{s} \cdot \boldsymbol{y}_{k}^{S}+\left(1-\lambda_{s}\right) \cdot \boldsymbol{y}_{k}^{N},
$$

where $\lambda_{s}$ is empirically set as 0.5 .

FH-GAT aggregates heterogeneous neighbors separately in each feature field with different field-specific attention, which delicately encodes user diverse preferences related to specific fields to the final node representation. Other GNN models could also be easily adapted to our framework. Comparing with other heterogeneous GAT models like [41], FH-GAT is more like a multi-channel aggregation, which collects field-specific user preferences in categories from multi-hop neighbors for node aggregation. These aggregated node embeddings are regarded as the final representations for all types of nodes in both offline training and online matching.

\subsection{Diversity-enhanced Training Objective}

Conventional ranking models usually rely on supervised training with CTR-oriented objectives, which also brings in homogenization. In this work, instead of merely focusing on CTR, GraphDR aims to learn user diverse preferences from multi-aspect factors and improve both accuracy and diversity. Therefore, we conduct the neighbor-similarity based loss [24] instead of conventional CTR-oriented objectives to highlight diversity. Specifically, we assume that all nodes should be similar to their neighbors on the diversified preference network regardless of their types. The neighborsimilarity based loss can be viewed as a specialized DeepWalk [30] with the path length set to be 2 (too long paths may bring in more noises and computation costs), which is formalized as follows:

$$
J=\sum_{h_{k}} \sum_{h_{i} \in N_{k}} \sum_{h_{j} \notin N_{k}}\left(\log \left(\sigma\left(\boldsymbol{h}_{k}^{\top} \boldsymbol{h}_{j}\right)\right)-\log \left(\sigma\left(\boldsymbol{h}_{k}^{\top} \boldsymbol{h}_{i}\right)\right)\right) .
$$

$\boldsymbol{h}_{k}$ is the $k$-th aggregated node embedding given by FH-GAT, and $N_{k}$ is the neighbor set of the k-th node. $\sigma(\cdot)$ indicates the sigmoid function. We use Adam [18] with negative sampling for training.

The feasibility and necessity of the neighbor-similarity based loss are discussed as follows: (1) videos that a user may be interested in are very likely to be connected via (multi-step) paths in the diversified preference network. For example, the multi-step path video:Apple event $\leftrightarrow$ tag:iPhone $\leftrightarrow$ tag:fast charge $\leftrightarrow$ video:new tech of charge connects two related videos users may watch sequentially. Through the neighbor-similarity based loss, related heterogeneous nodes linked by multi-hop paths in the diversified preference network will have similar representations. (2) GraphDR focuses on the matching module which values efficiency. Hence, the online multichannel matching in Sec. 4 conducts an embedding-based retrieval to meet the requirement of efficiency, which ranks videos according to the similarities between different types of embeddings. The neighbor-similarity based loss perfectly matches the embeddingbased retrieval for efficient, accurate and diverse matching.

Cooperating with diversified preference network, the neighborsimilarity based loss can well balance both accuracy and diversity, since it calculates video similarities with multiple factors including user watching habit in session, audience community, video content, taxonomy, and content provider. Precisely, the click-based supervised information used in classical ranking models is collected by two global interactions in GraphDR: video-video edges (for sequential click information in session) and video-user edges (for community-aggregated user-item interactions). These two types of click-based interactions are still the dominating interactions (taking nearly $83 \%$ of all interactions in our dataset given in Table 1 to ensure the recommendation accuracy. In contrast, the other four interactions related to tags, medias and words mainly provide the generalization ability of node representations to ensure the recommendation diversity. Comparing with classical CTR-oriented losses that merely focus on clicks, GraphDR jointly considers user diverse preferences from multiple heterogeneous interactions, and thus could achieve better accuracy and diversity in matching.

\section{ONLINE SERVING}

We have deployed our GraphDR on the matching module of a widely-used video recommendation system in WeChat Top Stories, which has nearly billion-level daily views generated by million-level users. We introduce the details of online serving.

\subsection{Online Multi-channel Matching}

Online multi-channel matching aims to retrieve hundreds of items from millions of candidates rapidly. GraphDR first builds the user representation with his/her valid watching behaviors $\left\{\hat{v}_{1}, \cdots, \hat{v}_{m}\right\}$ of videos. To improve the diversity, we conduct a multi-channel matching strategy as in Fig 2, which jointly retrieves video candidates from multiple aspects of representative tags, medias and videos in user historical behaviors.

In the video channel, each video in the valid watching behavior sequence retrieves top 100 videos with the cosine similarity between two aggregated video embeddings. The weighting score of the i-th video $v_{i}$ in the video channel is formulated as:

$$
\text { score }_{i}^{v}=\sum_{j=1}^{m} x_{v}(i j) \times \text { complete }_{j} \times \text { time }_{j} \times \operatorname{sim}\left(v_{i}, \hat{v}_{j}\right) .
$$

$x_{v}(i j)$ equals 1 only if the i-th video $v_{i}$ is in the top 100 nearest videos of the $j$-th video $\hat{v}_{j}$ in valid watching sequence, and otherwise equals 0 . complete $j_{j}$ is the watching time length percentage of $\hat{v}_{j}$, which measures the user's satisfaction of $\hat{v}_{j} . \operatorname{sim}\left(v_{i}, \hat{v}_{j}\right)$ represents the cosine similarity calculated by the aggregated node embeddings of $v_{i}$ and $\hat{v}_{j}$. We also use time $e_{j}$ to highlight the short-term interests 
of users as follows:

$$
\text { time }_{j}=\eta \cdot \text { time }_{j+1}, \quad \text { time }_{m}=1,
$$

in which $\eta=0.95$ is a time decay factor.

In the tag and media channels, we first learn user preferences on tags and medias from user historical behaviors. For example, the i-th tag's preference score $p_{i}^{t}$ is defined as:

$$
p_{i}^{t}=\sum_{j=1}^{m} z_{t}(i j) \times \text { complete }_{j} \times \text { time }_{j},
$$

where $z_{t}(i j)$ equals 1 when the $\mathrm{i}$-th tag belongs to $\hat{v}_{j}$, and otherwise equals 0 . To reduce noises, we only select top 10 tags $\hat{t}_{j}$ ranked by $p_{i}^{t}$ to form the user preferred tag set $T_{u}$. Next, each tag in $T_{u}$ retrieves top 100 videos with the cosine similarities between tag and video aggregated embeddings. The weighting score of the i-th video in tag channel is calculated as:

$$
\operatorname{score}_{i}^{t}=\sum_{\hat{t}_{j} \in T_{u}} x_{t}(i j) \times \frac{p_{j}^{t}}{\sum_{\hat{t}_{k} \in T_{u}} p_{k}^{t}} \times \operatorname{sim}\left(v_{i}, \hat{t}_{j}\right) .
$$

$x_{t}(i j)$ equals 1 if $v_{i}$ is in the top 100 nearest videos of $\hat{t}_{j}$, and otherwise equals $0 . \operatorname{sim}\left(v_{i}, \hat{t}_{j}\right)$ indicates the cosine similarity between $v_{i}$ and $\hat{t}_{j}$. The weighting score of $v_{i}$ in media channel score ${ }_{i}^{m}$ is calculated similarly as score ${ }_{i}^{t}$ of tag channel.

Finally, we combine all three multiple channels in the joint ranking to get the final video weighting scores as follows:

$$
\text { score }_{i}=\lambda_{v} \cdot \operatorname{score}_{i}^{v}+\lambda_{t} \cdot \operatorname{score}_{i}^{t}+\lambda_{m} \cdot \operatorname{score}_{i}^{m} .
$$

We rank all videos with their final video weighting scores and select top 500 videos as the output of GraphDR. We do not use the user group embedding learned by FH-GAT for online matching, since they are coarse-grained user community representations, and user historical behaviors are more informative for individuals. We also abandon the word channel considering the ambiguity in words.

\subsection{Online Deployment}

The online recommendation system mainly contains two modules including ranking and matching. The ranking module adopts ensemble ranking models including DeepFM [10], AutoInt [36] and AFN [5] to model feature interactions between user, item and contexts. Reinforcement learning is also used for long-term and list-wise rewards. In contrast, the matching module aims to retrieve as many appropriate items as possible. Therefore, the matching module consists of dozens of different types of matching strategies from various aspects. Our GraphDR and other compared matching baselines are worked as one of the matching strategies in the matching module. All matching strategies compete with each other, aiming to generate items to be fed into the the same shared ranking module. Sec. 5.6 gives the implementation details of an online matching evaluation.

Online matching especially values efficiency. In GraphDR, all embedding similarities like $\operatorname{sim}\left(v_{i}, \hat{v}_{j}\right)$ are pre-calculated in offline, which enables fast retrieval. Its online time complexity is less than $O(\log n)$ w.r.t the corpus size $n$, which is much superior to most deep ranking models involving complicated user-item interactions.

\section{EXPERIMENTS}

In experiments, we conduct extensive offline and online evaluations with detailed analyses on a real-world recommendation system to verify that GraphDR can improve both accuracy and diversity. In this section, we attempt to answer the following five research questions: (RQ1): How does the proposed GraphDR model perform against different types of competitive models on recommendation accuracy in matching (see Sec. 5.4)? (RQ2): How does GraphDR perform against competitive baselines on recommendation diversity at element level, list level and global level (see Sec. 5.5)? (RQ3): How does PAPERec perform in online system with various online accuracy and diversity related evaluation metrics (see Sec. 5.6)? (RQ4): How do different essential parameters affect GraphDR on recommendation accuracy and diversity (see Sec. 5.7)? (RQ5): Will node representations learned by GraphDR be successfully encoded with user diverse preferences (see Sec. 5.8)?

\subsection{Datasets}

Since there are few large-scale datasets for evaluating recommendation accuracy and diversity in matching, we build a novel dataset DivMat-2.1B extracted from WeChat Top Stories. We randomly select nearly 15 million users, collect their 2.1 billion video watching instances after data masking for privacy, and split the dataset into a train set and a test set using the chronological order. In train set, we build a huge diversified preference network following Sec. 3.2, where 15 million users are aggregated into 93 thousand user groups (users in the same user group have the same gender-age-location attribute triplet). The test set contains $8,132,719$ valid watching behavior instances for offline evaluation in matching.

Table 1: Statistics of the DivMat-2.1B dataset.

\begin{tabular}{c|c|c|c|c|c}
\hline video & user & tag & media & word & instance \\
\hline $1.2 \mathrm{M}$ & $15 \mathrm{M}$ & $103 \mathrm{~K}$ & $74 \mathrm{~K}$ & $150 \mathrm{~K}$ & $2.1 \mathrm{~B}$ \\
\hline \hline$\# \mathrm{v}-\mathrm{v}$ & $\# \mathrm{v}-\mathrm{t}$ & $\# \mathrm{v}-\mathrm{m}$ & $\# \mathrm{v}-\mathrm{w}$ & $\# \mathrm{v}-\mathrm{u}$ & $\# \mathrm{t}-\mathrm{t}$ \\
\hline $97 \mathrm{M}$ & $6.1 \mathrm{M}$ & $1.2 \mathrm{M}$ & $8.1 \mathrm{M}$ & $2.3 \mathrm{M}$ & $5.3 \mathrm{M}$ \\
\hline
\end{tabular}

\subsection{Competitors}

We implement several classical models as baselines, and categorize these competitors into four groups.

IR-based Methods. We implement three IR-based methods including Category-based, Tag-based and Media-based IR methods [17]. For Tag-based method, we build a tag-video inverted index, where videos are ranked with their popularity. The online matching retrieves videos with user preferred tags. Other IR-based methods are similar to Tag-based IR method.

CF-based Methods. We implement Item-CF [34] to retrieve similar videos with video co-occurrence. Moreover, we also implement BERT-CF, which uses semantic similarity to measure video similarity. Precisely, we calculate the semantic similarity of two videos with their title embeddings learned by BERT [7], and conduct CF to learn video embeddings for fast retrieval. 
Homogeneous NRL Methods. We implement some typical NRL models on the homogeneous video network built with video sessions. The compared methods include DeepWalk [30] and GraphSAGE [11]. These learned video representations are then used for online embedding-based matching with the video channel.

Neural-based Methods. Youtube candidate generation model [6] is a classical deep model for matching. We further improve the original Youtube model with behavior-level attention [52] and neural FM [12] as Youtube+ATT+FM, which is a strong industrial baseline in practice. Moreover, we implement DSSM [15], which retrieves items according to the user-item similarities. We also implement AutoInt [36] to model feature interactions. These models are optimized under supervised learning with video behaviors.

We conduct a nearest neighbor server for all embedding-based fast retrieval. Note that we do not compare with complicated diversified recommendation models specially designed for ranking, due to their tremendous computation costs in matching [16]. We do not report TDM/JDM either for the static tree-based retrieval is challenging to handle various aspects of diversities in videos.

Ablation Test Settings. We implement the heterogeneous versions of GraphSAGE [11] and GAT [38] to replace FH-GAT in the NRL module for ablation tests. We use GraphDR(GraphSAGE) and GraphDR(GAT) to represent these two settings respectively.

\subsection{Experimental Settings}

In GraphDR, the node feature embedding dimension is 900 , where the video field's dimension $d_{v}$ is 300 and others' are 150 . The dimensions of two output embeddings in FH-GAT are 120. The numbers of neighbor sampling in the first and second layers are 30 and 20. In training, we randomly select 20 negative samples for each positive sample, and set batch size as 512 . In online matching, we consider top 200 recent watched videos and retrieve top 500 candidates for ranking. The weighting scores $\lambda_{v}, \lambda_{t}$ and $\lambda_{m}$ are equally set to be 1 . We conduct the grid search for parameter selection. For fair comparisons, all models follow the same settings in evaluation.

\subsection{Recommendation Accuracy (RQ1)}

We first evaluate all GraphDR models and baselines on recommendation accuracy in offline DivMat-2.1B dataset.

5.4.1 Evaluation Protocols. We focus on matching that aims to generate hundreds of item candidates. Differing from ranking, matching only cares whether good items are retrieved, not the specific item ranks. Therefore, we use hit rate (HIT@N) [37] as the evaluation metric for accuracy, where an instance is "hit" if the clicked item is ranked in top N. We do not use classical ranking metrics such as MAP and NDCG since matching does not care specific ranks. To simulate the real-world scenarios, we conduct HIT@N with N set as 100, 200, 300 and 500. Since we retrieve top $\mathbf{5 0 0}$ items in the online recommendation system, HIT@500 is considered to be the most essential accuracy metric.

5.4.2 Experimental Results. In Table 2 we can observe that:

(1) GraphDR(FH-GAT) significantly outperforms all baselines on HIT@500 with the significance level $\alpha=0.01$. It indicates that GraphDR(FH-GAT) could retrieve accurate items in matching. Differing from conventional CTR-oriented models, GraphDR considers
Table 2: Results of recommendation accuracy. We set $\mathrm{N}=500$ in the matching module of our online system.

\begin{tabular}{l|c|c|c|c}
\hline HIT@N & $\mathrm{N}=100$ & $\mathrm{~N}=200$ & $\mathrm{~N}=300$ & $\mathrm{~N}=500$ \\
\hline Category-based & 0.0010 & 0.0018 & 0.0021 & 0.0031 \\
Tag-based & 0.0157 & 0.0207 & 0.0240 & 0.0287 \\
Media-based & 0.0235 & 0.0297 & 0.0337 & 0.0383 \\
\hline BERT-CF & 0.0337 & 0.0469 & 0.0556 & 0.0669 \\
Item-CF & 0.0748 & 0.0904 & 0.1214 & 0.1459 \\
\hline DeepWalk & 0.0799 & 0.0998 & 0.1130 & 0.1340 \\
GraphSAGE & 0.0932 & 0.1242 & 0.1568 & 0.1862 \\
\hline DSSM & 0.1012 & 0.1326 & 0.1631 & 0.2031 \\
AutoInt & 0.1087 & 0.1488 & 0.1892 & 0.2401 \\
Youtube+ATT+FM & $\mathbf{0 . 1 3 9 2}$ & $\mathbf{0 . 1 8 9 2}$ & 0.2194 & 0.2549 \\
\hline GraphDR(GraphSAGE) & 0.1013 & 0.1442 & 0.1818 & 0.2372 \\
GraphDR(GAT) & 0.1088 & 0.1674 & 0.2108 & $\underline{0.2731}$ \\
GraphDR(FH-GAT) & $\underline{0.1241}$ & $\underline{0.1885}$ & $\mathbf{0 . 2 3 8 4}$ & $\mathbf{0 . 3 1 0 2}$ \\
\hline
\end{tabular}

user diverse preferences related to video session, community, taxonomy, semantics and provider, which makes the matching results more diversified. GraphDR is perfectly suitable for matching, since it concerns more about item coverage than their specific ranks.

(2) GraphDR(FH-GAT) performs comparable or slightly worse than Youtube+ATT+FM when $N$ is small. It is intuitive since the neighbor-similarity based loss should balance accuracy and diversity, which inevitably harms ranking accuracy (not matching). In contrast, Youtube is a strong supervised baseline that benefits from its CTR-oriented objective. However, it suffers from overfitting and homogenization, and thus performs much worse than GraphDR(FHGAT) when $\mathrm{N}$ grows bigger (which is the practical scenario). The diversity issue will be discussed in Sec. 5.5.

(3) Both IR-based methods and BERT-CF are not satisfactory. It indicates that the taxonomy and semantic similarities contribute less to accuracy compared to user behaviors. In contrast, Neural-based methods focus on CTR-oriented objectives and thus get better accuracies. However, they still perform worse than GraphDR, for they fail to consider heterogeneous interactions and thus lack coverage.

Ablation study. Among different GraphDR versions, we find that FH-GAT outperforms GAT and GraphSAGE. It confirms the power of field-specific aggregation in modeling user diverse preferences. Moreover, we further conduct an ablation test to verify that all different types of nodes are necessary for the diversified recommendation. For instance, the HIT@500 will drop to $29.31 \%$ if we wipe out all word nodes in DivMat-2.1B.

\subsection{Recommendation Diversity (RQ2)}

In this subsection, we evaluate all models on both individual diversity and aggregate diversity in recommendation with various evaluation metrics.

5.5.1 Evaluation Protocols. We conduct nine typical diversity metrics and group them into three classes, namely the element-level diversity, the list-level diversity and the global-level diversity. The former two diversities indicate the individual diversity, while the 
Table 3: Results of different evaluation metrics on recommendation diversity.

\begin{tabular}{|c|c|c|c|c|c|c|c|c|c|}
\hline \multirow{2}{*}{ Model } & \multicolumn{3}{|c|}{ Element-level diversity } & \multicolumn{3}{|c|}{ List-level diversity } & \multicolumn{3}{|c|}{ Global-level diversity } \\
\hline & $\operatorname{tag}$ & cate & media & $\operatorname{tag}$ & cate & media & coverage & long-tail & novelty \\
\hline Category-based & 17.64 & 1.00 & 13.15 & 206.98 & 4.26 & 98.76 & 0.0012 & 0.0836 & 0.0043 \\
\hline Tag-based & 24.39 & 1.91 & 12.20 & 346.42 & 23.31 & 315.48 & 0.0270 & 0.1432 & 0.0343 \\
\hline Media-based & 29.67 & 2.95 & 1.00 & 434.30 & 43.41 & 9.58 & 0.0309 & 0.1327 & 0.0543 \\
\hline BERT-CF & 26.29 & 2.27 & 11.06 & 387.45 & 30.52 & 207.41 & 0.3829 & 0.2631 & 0.5734 \\
\hline Item-CF & 31.86 & 3.66 & 11.47 & 499.42 & 55.42 & 234.31 & 0.1786 & 0.0000 & 0.3143 \\
\hline DeepWalk & 30.64 & 3.24 & 13.23 & 476.76 & 52.53 & 246.33 & 0.1642 & 0.0000 & 0.3821 \\
\hline GraphSAGE & 31.67 & 2.84 & 13.65 & 426.32 & 41.11 & 285.52 & 0.1806 & 0.0000 & 0.3532 \\
\hline DSSM & 25.15 & 2.13 & 13.94 & 363.41 & 29.65 & 211.32 & 0.1688 & 0.0525 & 0.2843 \\
\hline AutoInt & 26.31 & 2.41 & 13.21 & 372.31 & 32.12 & 242.31 & 0.1762 & 0.0612 & 0.2971 \\
\hline Youtube+ATT+FM & 31.22 & 2.79 & 12.83 & 457.15 & 41.93 & 217.67 & 0.1532 & 0.0734 & 0.3523 \\
\hline GraphDR(GraphSAGE) & 33.19 & 3.61 & 14.91 & 498.31 & 51.21 & 327.28 & 0.4892 & 0.2854 & 0.6742 \\
\hline GraphDR(GAT) & $\underline{34.77}$ & 3.79 & $\underline{15.34}$ & $\underline{516.93}$ & $\underline{56.62}$ & 358.82 & $\underline{0.4934}$ & $\underline{0.3242}$ & $\underline{0.7032}$ \\
\hline GraphDR(FH-GAT) & $\overline{37.15}$ & $\overline{3.96}$ & $\overline{16.43}$ & $\overline{538.32}$ & $\overline{63.41}$ & $\overline{379.12}$ & $\overline{0.5132}$ & $\overline{0.3678}$ & $\overline{0.7352}$ \\
\hline
\end{tabular}

latter diversity measures the aggregate diversity [21]. The elementlevel diversity focuses on the diversity in each element, such as the tag, category, media in IR-based methods and the embeddings in baselines. Precisely, we regard the average deduplicated tag/category/media numbers in top 20 videos retrieved by these elements as the element-level diversity. The list-level diversity measures diversity in recommended lists (top 500 items). We use the average deduplicated tag/category/media numbers in the final recommended lists as the list-level diversity [42, 57]. In the globallevel diversity, coverage indicates the percentage of items that could be recommended [16]. Long-tail indicates the percentage of long-tail items in all results (videos that have not been watched for 15 days are empirically viewed as the long-tail videos). Novelty represents the percentage of new items generated by this model that other models do not recommend [50].

5.5.2 Experimental Results. Table 3 shows the results of various diversity metrics, form which we can know that:

(1) GraphDR(FH-GAT) achieves the best performances in all diversity metrics. The improvement derives from all three modules: (i) in diversified preference network, the heterogeneous interactions store user diverse preferences on taxonomy, semantics, community, video session and provider to link similar videos via multi-hop paths. (ii) In NRL, FH-GAT and its neighbor-similarity based loss successfully encode user diverse preferences into node representations. (iii) In online matching, the multi-channel strategy retrieves items from tag/media/video aspects, which also amplifies diversity. In addition, GraphDR(GraphSAGE) and GraphDR(GAT) generally outperform all baselines but still inferior to GraphDR(FH-GAT). It reconfirms the power of FH-GAT in diversity.

(2) The element-level and list-level diversities indirectly measure the individual diversity with diversities in tag, category and media. We assume that more tags/medias/categories in recommended lists indicate a more diversified recommendation. We find that behaviorbased models like Youtube and GraphSAGE perform better than other baselines in individual diversities. Nevertheless, GraphDR has better results since it considers other types of interactions.
(3) The global-level diversity measures the aggregate diversity, where coverage, long-tail and novelty focus on different aspects. Behavior-based models only consider video watching behaviors, which are hard to handle long-tail and new items. In contrast, BERT-CF focuses on content similarity and achieves good aggregate diversity. Still, GraphDR considers user diverse preferences in various fields and achieves the best aggregate diversity.

\subsection{Online Evaluation (RQ3)}

The offline evaluation has verified the improvements of accuracy and diversity in matching module. We further conduct an online A/B test to evaluate GraphDR in real-world industrial-level scenarios.

5.6.1 Evaluation Protocols. We implement GraphDR on the matching module of WeChat Top Stories following Sec. 4. The original online matching model is an ensemble model containing multiple IR-based, CF-based and Neural-based methods in Sec. 5.2. We regard GraphDR as an additional matching channel to the existing online ensemble model, with the ranking module unchanged. All videos retrieved by different matching channels will jointly compete with each other in the following ranking module.

In online $\mathrm{A} / \mathrm{B}$ test, we focus on the following seven representative metrics to evaluate accuracy and diversity: (1) video views per capita (VV), (2) video watching time per capita (VWT/c), (3) video watching time per video (VWT/v), (4) page turns per capita (PT), (5) deduplicated impressed videos per capita (DIV), (6) watched tag per capita (Tag diver), and (7) watched category per capita (Cate diver). The former five metrics mainly measure accuracy, while the latter two measure diversity. We conduct the A/B test for 5 days with nearly 3.8 million users involved, and report the improvement percentages over the ensemble base model. The online evaluation can be viewed as an online ablation test.

5.6.2 Experimental Results. Table 4 shows the results of online evaluation with multiple metrics, from which we find that:

(1) All GraphDR models outperform the ensemble base model, among which GraphDR(FH-GAT) achieves the best performances 
Table 4: Online A/B test on recommendation accuracy and diversity in a real-world system.

\begin{tabular}{|c|c|c|c|c|c|c|c|}
\hline & VV & $\mathrm{VWT} / \mathrm{c}$ & $\mathrm{VWT} / \mathrm{v}$ & PT & DIV & Tag diver & Cate diver \\
\hline GraphSAGE & $+3.08 \%$ & $+6.20 \%$ & $+1.48 \%$ & $+4.66 \%$ & $+2.43 \%$ & $+6.24 \%$ & $+10.27 \%$ \\
\hline GraphDR(GraphSAGE) & $+4.37 \%$ & $+7.61 \%$ & $+1.68 \%$ & $+6.04 \%$ & $+3.97 \%$ & $+9.16 \%$ & $+12.42 \%$ \\
\hline GraphDR(GAT) & $+5.30 \%$ & $+9.36 \%$ & $+2.49 \%$ & $+6.07 \%$ & $+8.00 \%$ & $+12.81 \%$ & $+15.57 \%$ \\
\hline GraphDR(FH-GAT) & $+6.08 \%$ & $+10.79 \%$ & $+3.10 \%$ & $+6.10 \%$ & $+10.43 \%$ & $+14.68 \%$ & $+17.00 \%$ \\
\hline
\end{tabular}

in accuracy and diversity with the significance level $\alpha=0.01$. We have also passed the homogeneity test in online evaluation, which confirms that the system and traffic split are unbiased and the improvements are stable. It verifies the effectiveness of GraphDR in real-world scenarios. Moreover, the improvements from GraphSAGE to FH-GAT also imply the significances of FH-GAT.

(2) The significant improvements in the former five metrics reflect better accuracy. A better video view metric indicates that users are more willing to click videos, while a better video watching time indicates users are genuinely interested in their clicked videos. Moreover, the page turns and deduplicated impressed video metrics also reflect user experiences indirectly. Users will slide down and browse more videos if they are satisfied with the results.

(3) The average watched tags and categories measure the diversity. The better tag/category diversity derives from two factors: more diverse videos impressed to users, and better personalized results that attract users to watch more videos. These diverse items help us to explore users' potential interests and give surprising results, which could even contribute to the long-term performances.

\subsection{Model Analyses (RQ4)}

We conduct several analyses on different channels and user behavior sequence lengths to better understand GraphDR.

5.7.1 Analysis on Multi-channel Matching. In GraphDR, the online multi-channel matching module plays an important role in improving diversity. We evaluate the GraphDR(FH-GAT) on HIT@N and list-level diversity metrics with different channels individually. From Table 5 we find that: the video channel achieves better HIT@N results, since video embeddings are directly influenced by video watching behaviors. In contrast, the tag and media channels are more responsible for diversity. To balance both accuracy and diversity, we combine all three channels in GraphDR.

Table 5: Results of different matching channels.

\begin{tabular}{c|c|c|c|c}
\hline Channel & tag & media & video & joint \\
\hline HIT@100 & 0.1027 & 0.0934 & $\mathbf{0 . 1 3 2 3}$ & 0.1241 \\
HIT@200 & 0.1571 & 0.1497 & $\mathbf{0 . 1 9 4 3}$ & 0.1885 \\
HIT@300 & 0.2143 & 0.2032 & $\mathbf{0 . 2 5 1 2}$ & 0.2384 \\
HIT@500 & 0.2787 & 0.2583 & $\mathbf{0 . 3 3 1 2}$ & 0.3102 \\
\hline Tag diversity & $\mathbf{5 7 3 . 4 3}$ & 543.31 & 468.42 & 538.32 \\
Cate diversity & $\mathbf{7 1 . 3 1}$ & 68.32 & 53.63 & 63.41 \\
Media diversity & 387.48 & $\mathbf{4 0 1 . 5 8}$ & 344.32 & 379.12 \\
\hline
\end{tabular}

5.7.2 Analysis on Behavior Sequence Length. We also analyze the impacts of different behavior sequence lengths in online matching. In Table 6, as the behavior sequence length increases, HIT@N metrics achieve consistent improvements, while diversity metrics become slightly worse. It implies that considering user long-term preferences can better understand users in recommendation. However, user long-term preferences are more stable, which inevitably harm the diversity. In GraphDR, we set the length as 200 since the improvements in accuracy are more significant than diversity.

Table 6: Results of different user behavior lengths.

\begin{tabular}{c|c|c|c|c}
\hline Length & $\mathrm{m}=20$ & $\mathrm{~m}=50$ & $\mathrm{~m}=100$ & $\mathrm{~m}=200$ \\
\hline HIT@100 & 0.0791 & 0.0883 & 0.1072 & $\mathbf{0 . 1 2 4 1}$ \\
HIT@200 & 0.1237 & 0.1373 & 0.1653 & $\mathbf{0 . 1 8 8 5}$ \\
HIT@300 & 0.1742 & 0.1902 & 0.2114 & $\mathbf{0 . 2 3 8 4}$ \\
HIT@500 & 0.2393 & 0.2617 & 0.2763 & $\mathbf{0 . 3 1 0 2}$ \\
\hline Tag diversity & $\mathbf{5 5 6 . 1 2}$ & 552.22 & 547.43 & 538.32 \\
Cate diversity & $\mathbf{6 9 . 5 2}$ & 68.11 & 66.73 & 63.41 \\
Media diversity & $\mathbf{3 9 5 . 4 5}$ & 391.52 & 387.91 & 379.12 \\
\hline
\end{tabular}

\subsection{Case Study (RQ5)}

In GraphDR, user diverse preferences are encoded in node embeddings. We give some tags and their nearest tags to explicitly display the diversity in Table 7. The interest in Restaurant guide may expand to specific food like Foie gras and their stories like Food documentary. The nearest tags of El Nino phenomenon reflect the interests in nature and science. Users like iPhone 11 Pro Max may also seek information on its hardware, software, and discount information. These nearest tags reflect both similarities in semantics and user preferences, since the node representations are learned under the neighbor-similarity based objective with a diversified preference graph containing various heterogeneous feature interactions. Similar phenomenon can be found in other nodes.

Table 7: Examples of tags and their nearest tags.

\begin{tabular}{c|l}
\hline Tag & Nearest tags \\
\hline $\begin{array}{c}\text { Restaurant } \\
\text { guide }\end{array}$ & $\begin{array}{l}\text { Roasted goose; Food documentary; Melaleuca } \\
\text { cake; Foie gras; Hong Kong cuisine }\end{array}$ \\
\hline $\begin{array}{c}\text { El Nino } \\
\text { phenomenon }\end{array}$ & $\begin{array}{l}\text { Superluminal speed; Easter island; Darwin; Abso- } \\
\text { lute zero; Parallel worlds theory }\end{array}$ \\
\hline $\begin{array}{c}\text { iPhone 11 Pro } \\
\text { Max }\end{array}$ & $\begin{array}{l}\text { iPhone SE; Fast charge; Mobile phone test; Voice } \\
\text { assistant; iPhone discount }\end{array}$ \\
\hline
\end{tabular}


Table 8 shows the nearest tags of some typical user groups. According to the node embeddings and aggregated behaviors, young men users in our dataset are more interested in sports, while young women focus more on fashion. Differing from the youth, the elderly in Beijing concentrate on traditional Chinese art and culture. The geographic distance also leads to fine-grained differences in interested sports (e.g., golf V.S. soccer). The preference divergences in different communities verify the success of diversity modeling.

Table 8: Examples of user groups with nearest tags.

\begin{tabular}{c|c|c|l}
\hline Sex & Age & City & Nearest tags \\
\hline$M$ & 21 & Beijing & $\begin{array}{l}\text { Sports news; Entrepreneur; Comedy; Scien- } \\
\text { tific anecdotes; Soccer }\end{array}$ \\
\hline F & 21 & Beijing & $\begin{array}{l}\text { Summer wear; Constellation; Product pro- } \\
\text { motion; Diet food; Potted plant }\end{array}$ \\
\hline$M$ & 59 & Beijing & $\begin{array}{l}\text { Calligraphy; Social documentary; Tai Chi; } \\
\text { Exercise; Family }\end{array}$ \\
\hline$M$ & 21 & London & $\begin{array}{l}\text { London Olympics; The Celtic; Scientists; } \\
\text { Golf; 100 metres race }\end{array}$ \\
\hline
\end{tabular}

\section{CONCLUSION AND FUTURE WORK}

In this work, we propose a simple and effective GraphDR framework to improve both accuracy and diversity in matching. We propose a new diversified preference network to capture heterogeneous interactions between essential objects in recommendation. We also design a novel FH-GAT model with a neighbor-similarity based loss to encode user diverse preferences from heterogeneous interactions. In experiments, we conduct extensive offline and online evaluations, model analyses and case studies. The significant improvements verify the effectiveness and robustness of GraphDR in improving accuracy and diversity simultaneously.

In the future, we will explore more types of interactions and weighted edges in GraphDR. Moreover, we will enhance the multichannel matching with more sophisticated models. Better graph neural networks are also worth being studied, which will be easily adopted in our GraphDR framework.

\section{REFERENCES}

[1] Tevfik Aytekin and Mahmut Özge Karakaya. 2014. Clustering-based diversity improvement in top-N recommendation. Journal of Intelligent Information Systems (2014).

[2] Keith Bradley and Barry Smyth. 2001. Improving recommendation diversity. In Proceedings of AICS

[3] Laming Chen, Guoxin Zhang, and Eric Zhou. 2018. Fast greedy map inference for determinantal point process to improve recommendation diversity. In Proceedings of NIPS.

[4] Heng-Tze Cheng, Levent Koc, Jeremiah Harmsen, Tal Shaked, Tushar Chandra, Hrishi Aradhye, Glen Anderson, Greg Corrado, Wei Chai, Mustafa Ispir, et al. 2016. Wide \& deep learning for recommender systems. In Proceedings of the 1st workshop on deep learning for recommender systems.

[5] Weiyu Cheng, Yanyan Shen, and Linpeng Huang. 2020. Adaptive Factorization Network: Learning Adaptive-Order Feature Interactions. In Proceedings of AAAI.

[6] Paul Covington, Jay Adams, and Emre Sargin. 2016. Deep neural networks for youtube recommendations. In Proceedings of RecSys.

[7] Jacob Devlin, Ming-Wei Chang, Kenton Lee, and Kristina Toutanova. 2019. Bert: Pre-training of deep bidirectional transformers for language understanding. In Proceedings of NAACL.
[8] Wenqi Fan, Yao Ma, Qing Li, Yuan He, Eric Zhao, Jiliang Tang, and Dawei Yin. 2019. Graph Neural Networks for Social Recommendation. In Proceedings of $W W W$.

[9] Lu Gan, Diana Nurbakova, Léa Laporte, and Sylvie Calabretto. 2020. Enhancing Recommendation Diversity using Determinantal Point Processes on Knowledge Graphs. In Proceedings of SIGIR.

[10] Huifeng Guo, Ruiming Tang, Yunming Ye, Zhenguo Li, and Xiuqiang He. 2017. DeepFM: a factorization-machine based neural network for CTR prediction. In Proceedings of IFCAI.

[11] Will Hamilton, Zhitao Ying, and Jure Leskovec. 2017. Inductive representation learning on large graphs. In Proceedings of NIPS.

[12] Xiangnan He and Tat-Seng Chua. 2017. Neural factorization machines for sparse predictive analytics. In Proceedings of SIGIR.

[13] Xiangnan He, Kuan Deng, Xiang Wang, Yan Li, Yongdong Zhang, and Meng Wang. 2020. LightGCN: Simplifying and Powering Graph Convolution Network for Recommendation. In Proceedings of SIGIR.

[14] Jui-Ting Huang, Ashish Sharma, Shuying Sun, Li Xia, David Zhang, Philip Pronin, Janani Padmanabhan, Giuseppe Ottaviano, and Linjun Yang. 2020. Embeddingbased retrieval in facebook search. In Proceedings of KDD.

[15] Po-Sen Huang, Xiaodong He, Jianfeng Gao, Li Deng, Alex Acero, and Larry Heck. 2013. Learning deep structured semantic models for web search using clickthrough data. In Proceedings of CIKM.

[16] Mahmut Özge Karakaya and Tevfik Aytekin. 2018. Effective methods for increasing aggregate diversity in recommender systems. knowledge and Information Systems (2018).

[17] Mohamed Koutheaïr Khribi, Mohamed Jemni, and Olfa Nasraoui. 2008. Automatic recommendations for e-learning personalization based on web usage mining techniques and information retrieval. In Proceedings of ICALT.

[18] Diederik P Kingma and Jimmy Ba. 2015. Adam: A method for stochastic optimization. In Proceedings of ICLR.

[19] Thomas N Kipf and Max Welling. 2017. Semi-supervised classification with graph convolutional networks. In Proceedings of ICLR.

[20] Yehuda Koren, Robert Bell, and Chris Volinsky. 2009. Matrix factorization techniques for recommender systems. Computer (2009).

[21] Matevž Kunaver and Tomaž Požrl. 2017. Diversity in recommender systems-A survey. Knowledge-Based Systems (2017).

[22] Shuai Li, Alexandros Karatzoglou, and Claudio Gentile. 2016. Collaborative filtering bandits. In Proceedings of SIGIR.

[23] Bin Liu, Chenxu Zhu, Guilin Li, Weinan Zhang, Jincai Lai, Ruiming Tang, Xiuqiang $\mathrm{He}$, Zhenguo Li, and Yong Yu. 2020. Autofis: Automatic feature interaction selection in factorization models for click-through rate prediction. In Proceedings of $K D D$.

[24] Qi Liu, Ruobing Xie, Lei Chen, Shukai Liu, Ke Tu, Peng Cui, Bo Zhang, and Leyu Lin. 2020. Graph Neural Network for Tag Ranking in Tag-enhanced Video Recommendation. In Proceedings of CIKM.

[25] Yong Liu, Yinan Zhang, Qiong Wu, Chunyan Miao, Lizhen Cui, Binqiang Zhao, Yin Zhao, and Lu Guan. 2019. Diversity-Promoting Deep Reinforcement Learning for Interactive Recommendation. arXiv preprint arXiv:1903.07826 (2019).

[26] Yuanfu Lu, Ruobing Xie, Chuan Shi, Yuan Fang, Wei Wang, Xu Zhang, and Leyu Lin. 2020. Social influence attentive neural network for friend-enhanced recommendation. In Proceedings of ECML-PKDD.

[27] Kanak Mahadik, Qingyun Wu, Shuai Li, and Amit Sabne. 2020. Fast distributed bandits for online recommendation systems. In Proceedings of ICS.

[28] Qiaozhu Mei, Jian Guo, and Dragomir Radev. 2010. Divrank: the interplay of prestige and diversity in information networks. In Proceedings of KDD.

[29] Sharad Nandanwar, Aayush Moroney, and M Narasimha Murty. 2018. Fusing Diversity in Recommendations in Heterogeneous Information Networks. In Proceedings of WSDM.

[30] Bryan Perozzi, Rami Al-Rfou, and Steven Skiena. 2014. Deepwalk: Online learning of social representations. In Proceedings of KDD.

[31] Lijing Qin and Xiaoyan Zhu. 2013. Promoting diversity in recommendation by entropy regularizer. In Twenty-Third International foint Conference on Artificial Intelligence.

[32] Yanru Qu, Han Cai, Kan Ren, Weinan Zhang, Yong Yu, Ying Wen, and Jun Wang. 2016. Product-based neural networks for user response prediction. In Proceedings of ICDM.

[33] Steffen Rendle. 2010. Factorization machines. In Proceedings of ICDM.

[34] Badrul Munir Sarwar, George Karypis, Joseph A Konstan, John Riedl, et al. 2001. Item-based collaborative filtering recommendation algorithms.. In Proceedings of $W W W$.

[35] Ying Shan, T Ryan Hoens, Jian Jiao, Haijing Wang, Dong Yu, and JC Mao. 2016. Deep crossing: Web-scale modeling without manually crafted combinatorial features. In Proceedings of KDD.

[36] Weiping Song, Chence Shi, Zhiping Xiao, Zhijian Duan, Yewen Xu, Ming Zhang, and Jian Tang. 2019. Autoint: Automatic feature interaction learning via selfattentive neural networks. In Proceedings of CIKM. 
[37] Fei Sun, Jun Liu, Jian Wu, Changhua Pei, Xiao Lin, Wenwu Ou, and Peng Jiang 2019. BERT4Rec: Sequential Recommendation with Bidirectional Encoder Representations from Transformer. In Proceedings of CIKM.

[38] Petar Veličković, Guillem Cucurull, Arantxa Casanova, Adriana Romero, Pietro Lio, and Yoshua Bengio. 2018. Graph attention networks. In Proceedings of ICLR.

[39] Jizhe Wang, Pipei Huang, Huan Zhao, Zhibo Zhang, Binqiang Zhao, and Dik Lun Lee. 2018. Billion-scale commodity embedding for e-commerce recommendation in alibaba. In Proceedings of KDD.

[40] Ruoxi Wang, Bin Fu, Gang Fu, and Mingliang Wang. 2017. Deep \& cross network for ad click predictions. In Proceedings of $A D K D D$.

[41] Xiao Wang, Houye Ji, Chuan Shi, Bai Wang, Yanfang Ye, Peng Cui, and Philip S Yu. 2019. Heterogeneous Graph Attention Network. In Proceedings of WWW.

[42] Le Wu, Qi Liu, Enhong Chen, Nicholas Jing Yuan, Guangming Guo, and Xing Xie. 2016. Relevance meets coverage: A unified framework to generate diversified recommendations. TIST (2016)

[43] Qiong Wu, Yong Liu, Chunyan Miao, Binqiang Zhao, Yin Zhao, and Lu Guan. 2019. PD-GAN: Adversarial Learning for Personalized Diversity-Promoting Recommendation. In IFCAI.

[44] Shu Wu, Yuyuan Tang, Yanqiao Zhu, Liang Wang, Xing Xie, and Tieniu Tan. 2019. Session-based Recommendation with Graph Neural Networks. In Proceedings of AAAI.

[45] Jun Xiao, Hao Ye, Xiangnan He, Hanwang Zhang, Fei Wu, and Tat-Seng Chua 2017. Attentional factorization machines: Learning the weight of feature interactions via attention networks. In Proceedings of IfCAI.

[46] Ruobing Xie, Cheng Ling, Yalong Wang, Rui Wang, Feng Xia, and Leyu Lin. 2020 Deep Feedback Network for Recommendation. In Proceedings of I7CAI.

[47] Ruobing Xie, Zhijie Qiu, Jun Rao, Yi Liu, Bo Zhang, and Leyu Lin. 2020. Internal and Contextual Attention Network for Cold-start Multi-channel Matching in
Recommendation. In Proceedings of I7CAI.

[48] Chuxu Zhang, Dongjin Song, Chao Huang, Ananthram Swami, and Nitesh V Chawla. 2019. Heterogeneous Graph Neural Network. In Proceedings of KDD.

[49] Lin Zhang, Qiang Yan, Junqiang Lu, Yongqiang Chen, and Yi Liu. 2019. Empirica Research on the Impact of Personalized Recommendation Diversity. In Proceedings of HICSS.

[50] Mi Zhang and Neil Hurley. 2008. Avoiding monotony: improving the diversity of recommendation lists. In Proceedings of RecSys.

[51] Weinan Zhang, Tianming Du, and Jun Wang. 2016. Deep learning over multi-field categorical data. In European conference on information retrieval.

[52] Guorui Zhou, Xiaoqiang Zhu, Chenru Song, Ying Fan, Han Zhu, Xiao Ma, Yanghui Yan, Junqi Jin, Han Li, and Kun Gai. 2018. Deep interest network for click-through rate prediction. In Proceedings of KDD.

[53] Han Zhu, Daqing Chang, Ziru Xu, Pengye Zhang, Xiang Li, Jie He, Han Li, Jian $\mathrm{Xu}$, and Kun Gai. 2019. Joint Optimization of Tree-based Index and Deep Model for Recommender Systems. In Proceedings of NIPS.

[54] Han Zhu, Xiang Li, Pengye Zhang, Guozheng Li, Jie He, Han Li, and Kun Gai. 2018. Learning Tree-based Deep Model for Recommender Systems. In Proceedings of $K D D$.

[55] Xiaojin Zhu, Andrew Goldberg, Jurgen Van Gael, and David Andrzejewski. 2007. Improving diversity in ranking using absorbing random walks. In Proceedings of NAACL.

[56] Jingwei Zhuo, Ziru Xu, Wei Dai, Han Zhu, Han Li, Jian Xu, and Kun Gai. 2020. Learning Optimal Tree Models under Beam Search. In Proceedings of ICML.

[57] Cai-Nicolas Ziegler, Sean M McNee, Joseph A Konstan, and Georg Lausen. 2005. Improving recommendation lists through topic diversification. In $W W W$. 